Received 00th January 20xx, Accepted 00th January 20xx

DOI: $10.1039 / \times 0 \times x 00000 x$ www.rsc.org/

\title{
QDs decorated with thiol-monomer ligands as new multicrosslinkers for the synthesis of smart luminescent nanogels and hydrogels
}

\begin{abstract}
M. Liras, ${ }^{a}$ I. Quijada-Garrido and O. García*
Herein new smart thermo-responsive fotoluminescent nano- and macrogel hybrids based on Quantum Dots (QDs) and poly(diethyelene glycol methyl ether methacrylate) ( $\left.\mathrm{MMEO}_{2} \mathrm{MA}\right)$ are reported. The synthetic stategy comprises the functionalization of the homemade hydrophobic CdSe nanoparticles (NPs) with thiol-functionalized methacrylic monomers by ligand exchange reaccions. Therefore, the organic-inorganic hybrid QDs NPs decorated with methacrylic groups are able to connect the inorganic core with the growing $\mathrm{pMEO}_{2} \mathrm{MA}$ chains during the radical polymerization reaction acting as multicrosslinker nanoagent. Depending if the bottom-up polymerization approach is done in solution or in bulk, nanometric core-shell QD@ $\mathrm{pMEO}_{2} \mathrm{MA}$ NPs or hybrid $\mathrm{pMEO}_{2} \mathrm{MA}$ hydrogels crosslinked by QD NPs are achieved. Both nanogel and hydrogel hybrids experience a considerable and sudden increase of the fluorescence emission upon heating, due to the gel collapse at the volume phase transition temperature (VPTT), independtly of $\mathrm{pH}$. Besides, at very basic $\mathrm{pH}$ $(\mathrm{pH} 12)$ the hydrogels also undergo a colour change from red to fully blue, which makes them promising as $\mathrm{pH}$ sensors. Both $\mathrm{pH}$ - and thermo-response are reversible in both nano- and macro-gel materials.
\end{abstract}

\section{Introduction}

Theranostic nanoplatfoms, combining therapeutic and diagnostic tool, are at the base of personalized medicine which main objective is to achieve the best therapeutic efficacy and minimizing the side effects. In this regard, the set-up of strategies to engineer nanoparticles combining multiple capabilities to apply in theranostic is a challenge. ${ }^{1-4}$ Among other nanosystems, multifunctional hybrid materials including high luminescent semiconductor Quantum Dots (QDs) ${ }^{5}$ are at the forefront of these novel multifunctional platforms given their unique optoelectrical and luminescence properties and they can be conveniently hybridized with polymers or bioactive molecules to obtain hybrid materials with unique functions and synergistic effect. ${ }^{6-8}$

Most of the recent advances focused in the development of these multifunctional systems are based on polymers as integrating factor. 8,9 Polymers can act as a compatible interphase between the NPs and the biological medium, facilitating their biotechnological implementation by the improvement on their solubility and dispersability, biocompatibility and stability in biological medium without to compromise their intrinsic properties. Moreover, the polymeric coating can also provide other properties and possibilities to the nanostructure such as functionalization, transport or controlled delivery of bioactive and therapeutic agents and drugs and/or response to external stimuli (temperature, $\mathrm{pH}$ or redox condition). ${ }^{1,8,10}$

Despite exciting progress in the development of functionalized metal nanoclusters or semiconductor nanoparticles a variety of challenges still remain and need to

\footnotetext{
a. Instituto de Ciencia y Tecnología de Polímeros (ICTP-CSIC).

C/ Juan de la Cierva, 3, E-28006-Madrid (Spain).

e-mail: ogarcia@ictp.csic.es

${ }^{b}$ Instituto IMDEA-Energía, Parque Tecnológico de Móstoles, Avda. Ramón de la Sagra, 3, E-28935 Móstoles-Madrid (Spain). + Electronic Supplementary Information (ES)
} DOI: $10.1039 / x 0 \times x 00000 x$ be addressed. Thus, new strategies to facilitate and improve the polymer-NP interactions are needed; much more taking into account factors as the number, the nature and the electronic properties of the reactive groups of the polymeric ligand in order to maintain or improve the intrinsic properties of the pristine NPs. Different ways as covalent and noncovalent interactions of nanoparticles with mono/multidentate polymers, encapsulation with amphiphilic or block copolymers or nanogels have been reported. ${ }^{2,9,11-14}$ Following this working line, in previous works, we explored the coating of plasmonic and luminescent nanoparticles with mono and multidentate methacrylic polymers with protected functional groups (thiol or amine). ${ }^{15-21}$ The new multifunctional copolymers endowed the new nanohybrids with long chemical and photophysical stability and an enhancement of their own pristine properties as well as the own polymeric starting characteristics as water solubility and even light, $\mathrm{pH}$ and temperature response. ${ }^{15-21}$ Up to now our NPs functionalization strategy is based on the synthesis of new methacrylic monomers with latent functional groups. The protected group, tioesther ${ }^{15,21}$ or boc-amino, ${ }^{19}$ allows the use of these new monomers by atom transfer radical polymerization process (ATRP) in order to obtain copolymers with controllable composition and structure. Taking into account this background, in this work the efficient coating of luminescent nanoparticles (Quantum Dots-QDs) with the new thiolated methacrylic monomers has been successfully carried out applying in situ ligand exchange reactions.

The new functionalized QD nanoparticles have been subsequently used as nanocrosslinking agents for the bottomup obtaining of new luminescent thermo-responsive hybrid nano- and macrogels based on $\mathrm{pMEO}_{2} \mathrm{MA}$, a methacrylate polymer with short oligo(ethylene oxide) side chains which is gaining attention in biomedical applications due to its water solubility, biocompatibility and nontoxicity. ${ }^{22-25}$

This is the first time, in our knowledge, that QDs have been available. See functionalized with thiolated methacrylic monomers making possible their use as nanocrosslinkers. The covalently 
integration of this hybrid QDs into three-dimensional nanoge and macrogel matrices will not only offer protection to QDs against different harsh chemical environments but also will provide unique photo-electronic properties to the resultant nanogels and nanocomposite hydrogels. This new approach could be useful for optoelectronics, catalysis and highthroughput assays for clinical applications as well as life science experiments; for example, in the development of systems capable of monitoring changes in temperature/pH in tumours tissues or as penetrating agent into the cell.

\section{Experimental Section}

\section{Materials}

The monomers, 2-(acetylthio) ethyl methacrylate (ACSEMA) and 2-(2-acetylthioethoxy) ethyl methacrylate (ACSEOMA), analogues of $\mathrm{MEO}_{2} \mathrm{MA}$ with a protected thiol group were synthesized according to the protocol previously described by our group. ${ }^{15,21}$ For the synthesis of the monomers, potassium thioacetate (Aldrich, 98\%), triethylamine (Aldrich, $\geq 99 \%), 2$ bromo-ethanol (Aldrich, 99\%) or 2-(2-chloroethoxy) ethanol (Aldrich, 99\%) and methacryloyl chloride (Aldrich, 99\%) were employed as received. For the CdSe nanocrystals, trioctylphosphine oxide (TOPO, 99\%, Aldrich), selenium powder (Aldrich), trioctylphosphine (TOP, Aldrich, 97\%), cadmium oxide brown (CdO, Fluka, 99\%), 1tetradecylphosphonic acid (TDPA, Alfa Aesar, 98\%) and sodium methoxide (Aldrich, 95\%) were used as received. Dialysis was carried out using a regenerated cellulose tubular membrane of MWCO 3500 Da from Cellu-Sep ${ }^{\circledR}$ T1. For the preparation of the nanogels and hydrogels, the monomer 2-(2-methoxyethoxy) ethyl methacrylate $\left(\mathrm{MEO}_{2} \mathrm{MA}\right.$, Aldrich, 95\%) was purified by passing through a neutral column to remove the antioxidant inhibitor; benzoyl peroxide (BPO) (Fluka, purum) and dimethyl aniline (DMA) freshly distilled (Fluka, > 98\%), $\mathrm{NaOH}$ (Panreac, $85 \%)$. Phosphate buffer solutions (PBS) of different pHs were prepared using sodium dihydrogen phosphate anhydrous (Fluka, $\geq 99 \%$ ), disodium hydrogen phosphate (Panreac, $\geq$ 98\%), orthophosphoric acid (Panreac, 85\%) and also sodium chloride (Panreac, $\geq 99.5 \%$ ) to keep constant the ionic strength $(\mu)$. Water was Milli $Q$ from water purification facility (millipore Milli-U10). Solvents were dried by standard methods or by elution through a Pure Solv Innovative Technology column drying system. Unless otherwise noted, reagents were commercially available and used without further purification.

\section{Synthesis of CdSe@TOPO nanoparticles (QDs NPs)}

Two batches of monodisperse and highly crystalline CdSe nanocrystals, with trioctylphosphine oxide (TOPO) as a ligand, were synthesized on a gram scale following a hightemperature procedure described in the literature. ${ }^{18,26}$ Firstly a $0.2 \mathrm{M}$ of selenium solution (Se) was prepared in a glove box by dissolving Se $(0.170 \mathrm{~g}, 2.15 \mathrm{mmol})$ in trioctylphosphine (TOP) $(10 \mathrm{~mL}, 21.5 \mathrm{mmol})$ until the solution was completely transparent. On the other hand, a mixture of cadmium oxide (CdO) (0.205 g, $1.6 \mathrm{mmol})$, TOPO (15.1 g, $39 \mathrm{mmol})$, and 1- tetradecylphosphonic acid (TDPA) $(0.9 \mathrm{~g}, 3.2 \mathrm{mmol})$ was continuously stirred and heated to $300^{\circ} \mathrm{C}$ under Argon flow until the dispersion turned to a transparent solution indicating that $\mathrm{Cd}$ was completely dissolved. Secondly, the Se solution in TOP was swiftly injected into this hot solution under vigorous stirring. After this nucleation step, reaction temperature was allowed to drop to $240^{\circ} \mathrm{C}$ to induce narrow growth of the CdSe nanocrystals. Once the desired size/colour (around $3 \mathrm{~nm}$ ) was reached, approximately 20-30 $\mathrm{min}$, the hot solution was poured into cold isopropanol, and the unused starting materials were separated from the nanocrystals synthesized by repeated decantation/centrifugation in isopropanol. The final product was dispersed in dichloromethane (DCM) and kept at $4^{\circ} \mathrm{C}$ in the dark under air for measurements and further applications.

Under these experimental conditions, two different $Q D$ nanoparticles were synthesized: $Q_{1}\left(3.3 \mathrm{~nm}, 6.6 \times 10^{-5} \mathrm{M}\right.$, $75860 \mathrm{~g} / \mathrm{mol})$ and $\mathrm{QD}_{2}\left(2.5 \mathrm{~nm}, 6.1 \times 10^{-5} \mathrm{M}, 32565 \mathrm{~g} / \mathrm{mol}\right)$. The size, molar concentration and the molecular weight of the obtained QDs were estimated as a function of their photophysical properties, as it was described by Yu et al. ${ }^{27}$

\section{Synthesis of hybrid nanoparticles by ligand exchange reaction}

In all ligand exchange reactions, $2 \mathrm{~mL}$ of the purified TOPO coated $Q D_{n}\left(Q D_{1}\right.$ or $\left.Q D_{2}\right)$ in dichloromethane were mixed with the proper quantity of ACSEMA or ACSEOMA monomer in order to obtain a 1/1000 molar ratio of [QD]/[thiol-monomer]. As an example, $25 \mathrm{mg}$ of AcSEMA $\left(6.6 \times 10^{-2} \mathrm{M}\right)$ were added to 2 $\mathrm{mL}$ of $\mathrm{QD}_{1} @ \mathrm{TOPO}\left(6.6 \times 10^{-5} \mathrm{M}\right)$ in dichloromethane (DCM). The reactions were maintained at room temperature for 2-5 days to ensure the total functionalization of the QD surface by the thioacetylated monomer, both in absence and presence of a of sodium methoxide $\left(\mathrm{CH}_{3} \mathrm{ONa}\right)$ (1-2 $\left.\mathrm{mg}\right)$. The reactions were monitored by FTIR-ATR and ${ }^{1} \mathrm{H}-\mathrm{NMR}$ (Figures S1, S2 and S3) in order to determine the efficiency on $Q D_{n}$ surface functionalization by the acetythiol (QD@AcSEO ${ }_{n} M A$ ) or thiol (QD@SEO $\left.{ }_{n} M A\right)$ groups from the monomers as well as their effect on the final emission properties of the hybrids. Afterwards, the crude product was placed inside a regenerated cellulose membrane tubing of MWCO-3500 Da and dialyzed against a solvent mixture tetrahydrofurane/water $(7 / 3 \mathrm{v} / \mathrm{v})$ (replacing fractions of $15 \mathrm{~mL}$ every 12-24 h) until no monomer was detected by UV spectrometry in the dialyzed fraction, usually after $45-60 \mathrm{~mL}$ of solvent. In the hybrids of QD@ACSEOnMA not yet hydrolysed, for the subsequent hydrolysis step, a little amount of sodium methoxide $\left(\mathrm{CH}_{3} \mathrm{ONa}\right)$ (1-2 mg) was added over a dialyzed solution in DCM under stirring at room temperature, usually 2 days. The hydrolysis reaction was monitored by FTIR-ATR (Figure S2). Excess of $\mathrm{CH}_{3} \mathrm{ONa}$ was removed washing with water the DCM solutions (Scheme 1).

The hybrid nanoparticles of $\mathrm{QD}_{1} @ S E M A$ and $\mathrm{QD}_{2} @ S E O M A$ were employed as multicrosslinker to obtain core-shell QDs@nanogels and QDs-hydrogel nanocomposites based on $\mathrm{pMEO}_{2} \mathrm{MA}$. 


\section{Synthesis of hybrid QD@SEOnMA@pMEO_MA nanogels}

Nanogels were synthesized by redox initiated free-radical random polymerization in solution. In all cases, polymerization mixtures with $1 / 50000$ molar ratio of $\left[\mathrm{QD} @ S E \mathrm{~S}_{\mathrm{n}} \mathrm{MA}\right] /\left[\mathrm{MEO}_{2} \mathrm{MA}\right]$ in dichloromethane were prepared including the initiator BPO (5 wt\%) and the activator DMA (1 wt\%) of the polymerization, both of them refers to the total monomer amount. The polymerization mixtures were maintained at room temperature and under vigorous stirring for two days. Afterwards, the samples were precipitated and washed in excess of hexane to remove the unreacted reagents (NPs, monomer and/or initiators). The fluorescent coloured precipitate was re-dissolved in THF and placed inside a regenerated cellulose membrane tubing of MWCO-50 kDa and dialyzed against water at $4^{\circ} \mathrm{C}$ in order to remove small oligomers as well as possible $\mathrm{pMEO}_{2} \mathrm{MA}$ homopolymer formed during the polymerization process, which is soluble in the aqueous medium.

\section{Synthesis of hybrid QD@SEO ${ }_{n}$ MA-pMEO 2 MA hydrogel nanocomposites}

The hydrogels were synthesized by redox initiated freeradical random bulk polymerization of $\mathrm{MEO}_{2} \mathrm{MA}$ in the presence of QD@SEO ${ }_{n} M A$ as Scheme 1 displays. In all cases, polymerization mixtures with total monomer amount of $1 \mathrm{~g}$ were prepared, including the initiator BPO (5 wt\%) and the activator DMA (1 wt\%) of the polymerization, both of them refers to the total monomer amount, to proceeded the reaction rapidly and at room temperature.

Six different compositions of hybrid hydrogels were assayed employing always the same majority monomer $\left(\mathrm{MEO}_{2} \mathrm{MA}\right)$ and different feed ratios of the multicrosslinker hybrid nanoparticles (1-3 wt\%) included in the polymerization mixture. The procedure to obtain sheet-shaped gels was as follows. The hybrid mixture solution was injected in moulds formed by two glass plates (previously silanised with dichlorodimethylsilane solution to avoid the adhesion of the gels and facilitate their subsequent demoulding) enclosed by a rubber framework/spacer of $500 \mu \mathrm{m}$ thickness in order to avoid air contact during the polymerization. After two hours the gelation was observed. However, to ensure the total reaction of the monomers, the system was allowed to react for at least 24 hours at room temperature. Afterwards, the ge sheets were removed from the glass plate and were cut into uniform films of about $10 \times 20 \times 0.5 \mathrm{~mm}$. The hydrogel films were then immersed in MilliQ water for at least 3 days to remove the unreacted chemicals. During this time the samples were maintained at $4{ }^{\circ} \mathrm{C}$ and the water was replaced several times. After that, the hydrogels were dried at room temperature until constant weight was reached.

A control assay with the same formulation mixture but in absence of multicrosslinked nanoparticles was done following the same synthetic procedure. The film formed was completely soluble in MilliQ water at $4^{\circ} \mathrm{C}$.

\section{Characterization}

UV-Vis absorption and fluorescence spectra were recorded on a Perkin-Elmer Lambda-35 and Perkin-Elmer LS5OB spectrophotometer, respectively. The fluorescence quantum yield $\left(\Phi_{\mathrm{F}}\right)$ (excitation at $450 \mathrm{~nm}$ for all samples except QD@SEOMA@pMEO 2 MA nanogels in DCM which was excited at $400 \mathrm{~nm}$ ) of the dilute dichloromethane solutions of QDs was evaluated relative to 4-(Dicyanomethylene)-2-methyl-6-(4dimethylamino styryl)-4H-pyran (DCMSP) dye in ethanol solution $\left(\Phi_{\mathrm{F}}=0.435\right)$ for $\mathrm{QD}_{1}{ }^{28}$ or to fluorescein in basic water $\left(\Phi_{\mathrm{F}}=0.92\right)$ for $\mathrm{QD}_{2}{ }^{29}$. Experiments of fluorescence emission as function of $\mathrm{pH}$ and temperature were done, choosing one temperature below, $4^{\circ} \mathrm{C}$, and one temperature above, $50^{\circ} \mathrm{C}$ of their volume phase transition temperature (VPTT) $\left(21-31^{\circ} \mathrm{C}\right)$. The experimental was carry out from the concentrated nanogels water solutions or immersing the hydrogel films in phosphate buffer solutions (PBS) prepared at different $\mathrm{pHs}$ $(\mathrm{pH}=2,4,5,7,9$ and 12$)$ keeping the ionic strength constant $(0.1 \mathrm{M})$. Samples were left to equilibrate at $4^{\circ} \mathrm{C}$ before recording their fluorescence emission spectra, after that, samples were equilibrated at $50^{\circ} \mathrm{C}$ and fluorescence emission spectra recorded and so on (excitation at $450 \mathrm{~nm}$ in all samples). The samples were thermostated using a Huberpolycat cc1 cryostat system or a Julabo-paratherm U5electronic thermostatic bath. In all cases the fluorescence signals were recorded in the front-face mode by orienting the immersed samples at angles of $35^{\circ}$ and $55^{\circ}$ with respect to the excitation and emission beams, respectively when the measures were done at different temperatures. The recorded fluorescence spectra were the average of at least three independent measurements made until reproducible fluorescence intensity was reached.

The volume phase transition temperature (VPTT) was studied by determining the cloud point temperature (Tc), by measuring the optical transmittance at $700 \mathrm{~nm}$ as a function of temperature and $\mathrm{pH}$. The analysis was made using a Cary 3 BIO-Varian UV-visible spectrophotometer equipped with a Peltier thermostatic accessory. The temperature was raised from 7 to $60^{\circ} \mathrm{C}$ above the transition at a rate of $1^{\circ} \mathrm{C} \mathrm{min}$. The cloud point is produced by the phase separation when the volume phase transition temperature (VPTT) is exceeded and was defined as the temperature at the inflection point of the absorbance versus temperature curve. Equilibrium swelling values $\left(Q_{\infty}\right)$ of the hydrogels were determined gravimetrically. Dried gels were left to swell in PBS or distilled water at different experimental conditions during 24 hours. Then, samples were taken out, wiped superficially with blotting paper and weighed. The $Q_{\infty}$ was calculated in grams of water per grams of dry gel using the following expression:

$$
Q_{\infty}=\left(m_{\infty}-m_{0}\right) / m_{0}
$$

where $m_{\infty}$ is the weight of the swollen gel at equilibrium, $m_{0}$ is the weight of the dry polymer gel. 


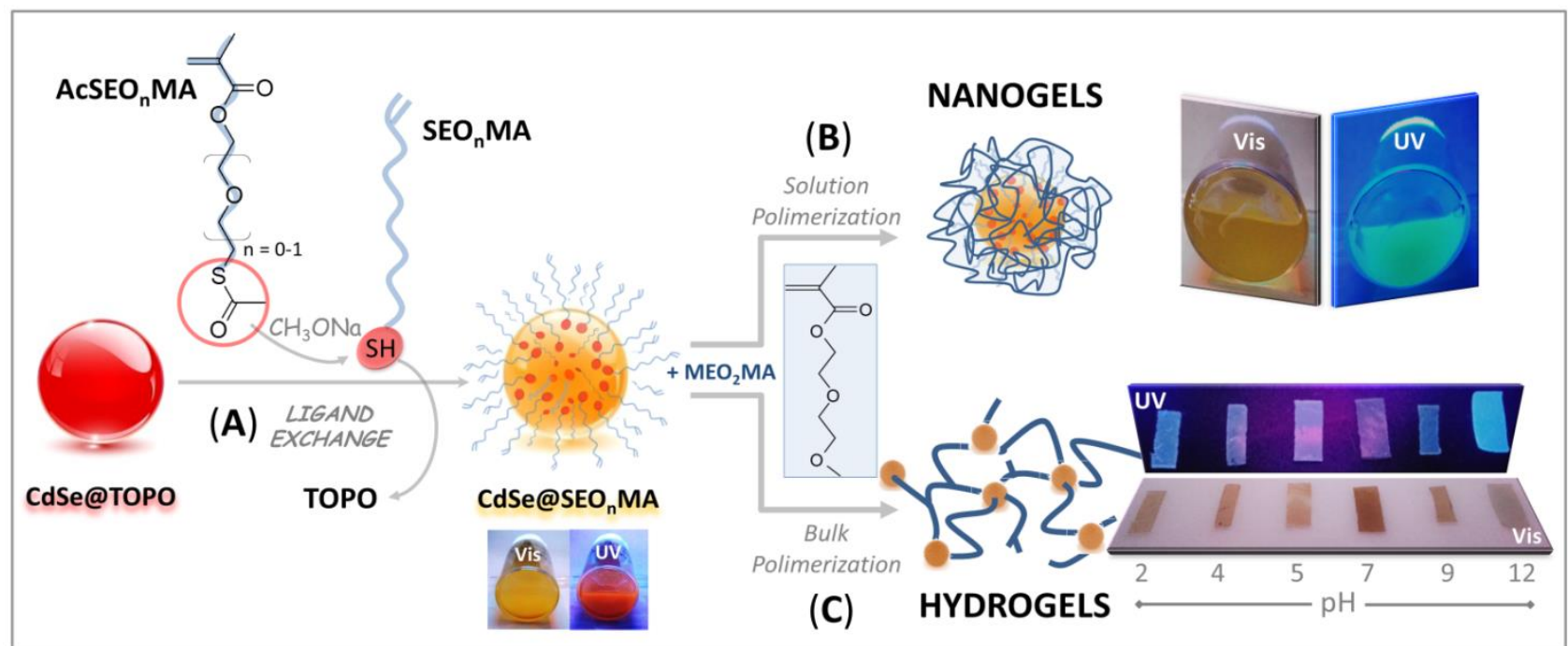

Scheme 1. Schematic representation of: (A) QDs (CdSe@TOPO) functionalization with thiolated monomers (AcSEO $\left.{ }_{n} M A\right)$ to form multicrosslinker QDs (CdSe@SEO $\left.{ }_{n} M A\right) ;(B)$ Synthesis of QD@SEO ${ }_{n} M A @ \mathrm{pMEO}_{2} \mathrm{MA}$ nanogels and UV/Vis photographs of the nanogels in organic medium; (C) Synthesis of hybrid hydrogels of pMEO ${ }_{2} \mathrm{MA}$ with QD@SEO $\mathrm{n}_{\mathrm{n}} \mathrm{MA}$ nanocrosslinker and photographs of the hybrid nanocomposite sheets swollen in aqueous solutions at different pHs illuminated under both visible and UV light.

Dynamic light scattering (DLS) measurements of the nanogels were performed using a Malvern Zetasizer Nano Z spectrometer with a $10 \mathrm{~mW}$ HeNe laser at $633 \mathrm{~nm}$. All measurements were performed at a scattering angle of $173^{\circ}$. All the solution samples were measured immediately after the dialysis with a concentration of $1 \mathrm{mg} \mathrm{mL}^{-1}$ in dichloromethane at $25^{\circ} \mathrm{C}$ or in aqueous buffer solutions at different $\mathrm{pHs}$ and temperatures, taking into account the corresponding viscosity and refractive index of the solvent in each case. The experimental data provided are the size distribution by number calculated from the autocorrelation function using Malvern's Zetasizer software from at least 10 different measurements.

Infrared spectra were obtained using a Spectrum One FTIR spectrometer (Perkin-Elmer) fitted with an attenuated total reflectance (ATR) accessory under unforced conditions. The samples were placed in direct contact with the diamond crystal without previous preparation. Measurements were collected at $6 \mathrm{~cm}^{-1}$ resolution and 4 scans per spectrum.

${ }^{1} \mathrm{H}-\mathrm{NMR}$ spectra were recorded on a Bruker $400 \mathrm{MHz}$ spectrometer in $\mathrm{CDCl} 3$ at room temperature.

Scanning transmission electron microscopy (sTEM) images were taken using a JEOL JEM-2100 microscopy operating at $200 \mathrm{kV}$ equipped with a charged-coupled device (CCD)-camera (Gatan Orius SC1000). Usually, for sample preparation, one drop of functionalized QDs $(\sim 5 \mu \mathrm{M})$ in dichloromethane solution was left to dry under air at room temperature over ultrathin carbon type-A film supported on a 400 mesh copper grid ( $3 \mathrm{~mm}$ in diameter) (from Ted Pella, Inc.). In order to determine the thermally induced aggregation of QD@nanogels by STEM, the preparation of the hybrid nanogels was done as follows: about 5-10 $\mu \mathrm{L}$ of a $1 \mathrm{mg} \mathrm{mL}^{-1}$ of the same aqueous solution of the hybrid nanogels were left to dry under vacuum or under hot air flow, on a carbon film supported on the same type of copper grid.

\section{Results and discussion}

Advanced progress of our recent research involves the surface functionalization of homemade $Q D s\left(Q D_{1}\right.$ and $\left.Q D_{2}\right)$ with both 2-(acetylthio) ethyl methacrytale (ACSEMA) and 2-(2(acetylthio)ethoxy) ethyl methacrylate (ACSEOMA), analogues of $\mathrm{MEO}_{2} \mathrm{MA}$ monomer having protected thiol group, in presence or not of sodium methoxide, in order to investigate: i) the influence of the ligand exchange reaction with an acetyl/thiolated small molecule on the photoluminescent properties of the QD; ii) the ability of QD@SEO ${ }_{n} M A$ to be used as multicrosslinker agent in the synthesis of new hybrid nanoand macro-gels and iii) the effect of the thiolated monomer length in the final properties of the hybrid materials.

The synthesis of the QDs functionalized with the new thiolated monomers was carried out by ligand exchange reactions. The procedure involved the mixture of homemade CdSe@TOPO with the thiol-monomer (AcSEMA or AcSEOMA) in a molar ratio of $1 / 1000$ in dichloromethane maintaining this solution under stirring at room temperature for 2-5 days in presence or in absence of sodium methoxide $\left(\mathrm{CH}_{3} \mathrm{ONa}\right)$, following the exchange of TOPO by the thiol-monomer by FTIR-ATR and ${ }^{1} \mathrm{H}-\mathrm{NMR}$ (Figure S1, S2 and S3). Under the $\mathrm{CH}_{3} \mathrm{ONa}$ basic medium, the thioacetate group is hydrolysed into thiol. This reaction was monitored by both FTIR-ATR (Figure S2) following the disappearance of the thioester band and by the shift of the proton signal corresponding to the $\mathrm{CH}_{2}$-S at ${ }^{1} \mathrm{H}-\mathrm{NMR}$ spectrum (Figure S3). Note that based on FTIR-ATR results the hydrolysis of the ester group to alcohol has been discarded because the carbonyl group is intact and the $-\mathrm{OH}$ band did not appeared. Result confirmed by ${ }^{1} \mathrm{H}-\mathrm{NMR}$ where the hydrolysed product does not appear. In a previous work $^{18}$ we reported that both the thioacetate as the thiol group have enough affinity by the CdSe surface to displace the TOPO. Therefore, initially two types of hybrids NPs were obtained: surface functionalized with thioacetate group 
( $Q D_{1} @ A c S E M A$ and $\left.\mathrm{QD}_{2} @ A c S E O M A\right)$ and surface functionalized with thiol groups $\left(\mathrm{QD}_{1} @ S E M A\right.$ and $\left.\mathrm{QD}_{2} @ S E O M A\right)$. In both cases the functionalization of QDs gives rise to new fluorescent nanohybrids which have a multiple methacrylate function around the QD core capable of act as a multicrosslinking agent in the polymerization processes, as detailed in Scheme 1. Note that due to the homemade origin of the pristine $Q D s$, two different batches $\left(Q_{1}\right.$ and $\left.Q D_{2}\right)$ were used for each kind of monomers, long and short, but as we will see below the general conclusions are independent of the nanoparticles size used.

The optical properties (absorption and emission) of the new CdSe@thiol-monomer nanoparticles are collected in Table 1 and S1 and displayed in Figure 1 and S4.

As it can be observed in Figure 1, absorption and emission maxima of the synthesized CdSe@thiol-monomers are similar to those of pristine CdSe@TOPO but a noticeable change of the fluorescence intensity is detected (summarized in Table 1 and Figure 1). While the functionalization with a small molecule with thioacetate pendant group produces an enhance of the fluorescence (Table S1) the functionalization with a thiol group causes a quenching of these fluorescence emission for the shorter monomer (Table 1, entry 2) or slightly improves this parameter for large monomer (Table 1, entry 5), but the results achieved are independent of whether hydrolysis to thiol group is carried out in a single step or in two stages.

In general, photoluminescence of QDs is strongly dependent on both the shell material and its thickness. ${ }^{30,31}$ These results are in accordance with those found by other authors for CdSe QDs functionalized with small monodentate thiols, such as 11-mercaptoundecanoic acid (MUA), and 3mercaptopropionic acid (MPA) ${ }^{32,33}$ and the usually red shift of the absorption and emission bands are observed with respect to the parent QD@TOPO. ${ }^{34}$ The quenching efficiency of the luminescence of the CdSe QDs by the ligand was found to depend strongly on both the size and charge of the thiol, and, for this reason, a stronger decrease of the luminescence is detected for the case of the shorter SEMA monomer than when a longer monomer as SEOMA is used, probably related with a suitable protected surface to the $Q D$ versus the environment.

In a second step, the hybrid nanoparticles obtained, QD@SEMA and QD@SEOMA, have been used as
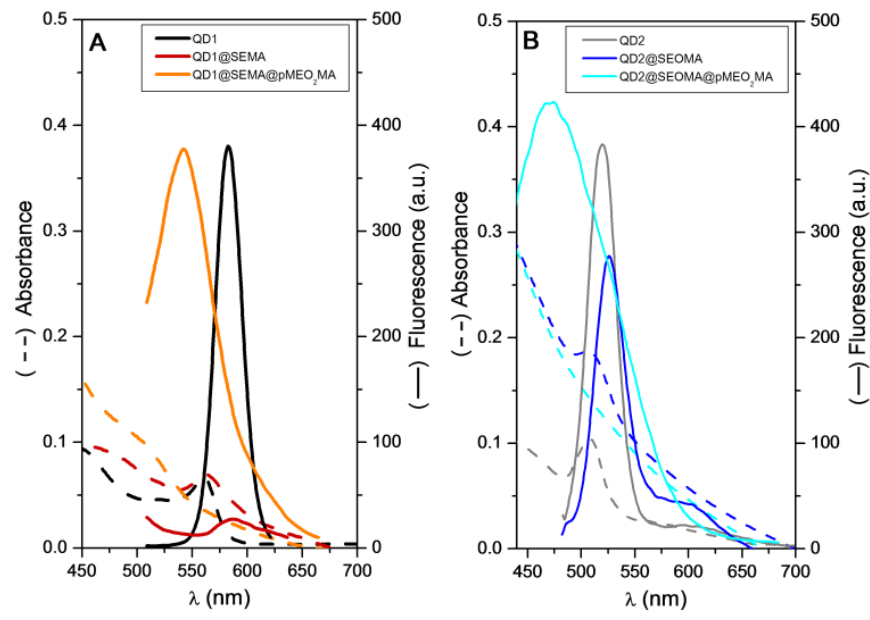

Figure 1. Absorption spectra (dashed lines) and fluorescence spectra (solid lines) of pristine CdSe@TOPO $\left(\mathrm{QD}_{\mathrm{n}}\right), \mathrm{QD} @ \mathrm{SEO}_{\mathrm{n}} \mathrm{MA}$ and QD@SEO $\mathrm{n}_{\mathrm{n}} \mathrm{MA} @ \mathrm{pMEO}_{2} \mathrm{MA}$ nanogels in dichloromethane solutions. (Excitation at $450 \mathrm{~nm}$ in all samples except QD@SEOMA@pMEO 2 MA nanogels in DCM which was excited at 400nm).

multicrosslinked agent in the synthesis of nanogels and hydrogels as it is represented in Scheme 1.

\section{Synthesis and characterization of hybrid thermo-responsive QD@nanogels}

As it was previously described in the experimental section, the methacrylate decorated QDs have been used as a core for the synthesis of QD@nanogels by the growth of a thin polymeric coating of $\mathrm{pMEO}_{2} \mathrm{MA}$ around the semiconductor nanoparticle. Nanogels were synthesized by redox initiated free-radical random polymerization in dichloromethane solution starting from mixtures of the multi-methacrylic functionalized QDs (QD@SEO $\left.{ }_{n} M A\right)$ and $\mathrm{MEO}_{2} \mathrm{MA}$ as comonomer. Under these experimental conditions the obtained hybrid QD@nanogels maintain their small hydrodynamic size in the order of few nanometres (Table 1).

Note that the reaction is done in the absence of additional crosslinker monomer or surfactants; in addition, when the polymerization of $\mathrm{MEO}_{2} \mathrm{MA}$ was carried out without the presence of QD@SEOnMA, linear soluble $\mathrm{pMEO}_{2} \mathrm{MA}$ chains were formed. These facts, corroborates the hypothesis that the multi-methacrylate funcionalized QD nanoparticles are necessary to obtain the core-shell QD@nanogels.

Table 1. Photophysical properties ${ }^{a}$ and nanoparticle sizes ${ }^{b}$ of the hybrids nanoparticles in dichloromethane solution.

\begin{tabular}{|c|c|c|c|c|c|c|}
\hline Entry & sample & $\begin{array}{c}\lambda_{\text {abs.max. }} \\
(\mathrm{nm})\end{array}$ & $\begin{array}{c}\lambda_{\text {em.max. }} \\
(n \mathrm{~nm})\end{array}$ & $\Phi_{\mathrm{F}}$ & ${ }^{\mathrm{c}} \Phi_{\mathrm{F}}$ rel. & $\begin{array}{l}\text { Size } \\
(\mathrm{nm})\end{array}$ \\
\hline 1 & $\mathbf{Q D}_{1}$ & 560.9 & 570.5 & 0.031 & 1 & $3.3^{*}$ \\
\hline 2 & QD $1 @ S E M A$ & 562.9 & 575.7 & 0.006 & 0.2 & $3.2 \pm 0.6$ \\
\hline 3 & QD $1 @ S E M A @ p M E O_{2} M A$ nanogels & 510.0 & 526.9 & 0.050 & 1.6 & $6.1 \pm 0.5$ \\
\hline 4 & $Q_{2}$ & 507.9 & 520.8 & 0.015 & 1 & $2.5^{*}$ \\
\hline 5 & QD $_{2} @ S E O M A$ & 505.6 & 527.6 & 0.018 & 1.2 & $3.6 \pm 0.6$ \\
\hline 6 & $\mathrm{QD}_{2} @ S E O M A @ p M E O_{2} \mathrm{MA}$ nanogels & $360-450$ & 470.0 & 0.018 & 1.2 & $8.0 \pm 1.1$ \\
\hline
\end{tabular}

${ }^{a}$ Maximum absorption wavelength $\left(\lambda_{\text {abs.max }}\right)$, maximum emission wavelength $\left(\lambda_{\text {em.max }}\right)$, emission quantum yield taking as reference DCMSP in ethanol $\left(\Phi_{\mathrm{F}}=0.435\right)$ for $\mathrm{QD}_{1}{ }^{28}$ or to fluorescein in basic water $\left(\Phi_{\mathrm{F}}=0.92\right)$ for $\mathrm{QD}_{2}{ }^{29}{ }$, ${ }^{\mathrm{c}}$ emission quantum yield related $\left(\Phi_{\mathrm{F} \text { rel. }}\right)$ to the parent CdSe@TOPO $\left(Q D_{\mathrm{n}}\right)\left(\Phi_{\mathrm{F} \text { rel }}\right) .{ }^{\mathrm{b}} \mathrm{Measured}$ by dynamic light scattering $(\mathrm{DLS})$ in diluted dichloromethane solutions. *Nanoparticle Sizes estimated as a function of their photophysical properties as it was described by Yu et al. ${ }^{27}$ 

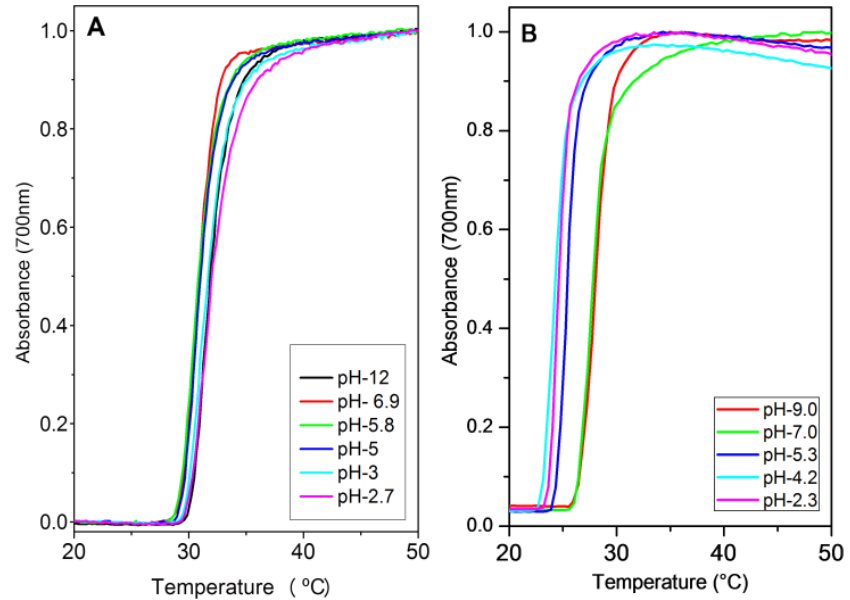

Figure 2. Normalized absorbance at $700 \mathrm{~nm}$ vs temperature for nanogels of $\mathrm{A}$ ) QD@SEMA@pMEO 2 MA and B) QD@SEOMA@pMEO 2 MA dissolved $\left(1 \mathrm{mg} \mathrm{mL}^{-1}\right)$ in buffer solutions of the $\mathrm{pHs}$ indicated in the Figure (from 12 to 2).

The photophysical properties of the hybrids, QD@nanogels, in dichloromethane solution are presented in Figure 1 and Table 1. As can be observed, the new hybrid QD@nanogels, do not exhibit a well-defined absorption peak due to the growth of the $\mathrm{MEO}_{2} \mathrm{MA}$ coating as shown in Figure 1 but the reduction in the luminescent quantum yield mediated by thiol binding is mitigated in the case of SEMA by the crosslinking of the polymeric shell around the functionalized QDs as other authors also found, ${ }^{35}$ while SEOMA based nanogels maintained their emission properties. On the other hand is clearly noticeable how hybrid nanogels emission features have experienced a strong hypsochromic shift, up to $50 \mathrm{~nm}$, with respect to both pristine QD@TOPO and QD@SEO ${ }_{n} M A$. The broadening of emission when the particles are coated by the $\mathrm{p}\left(\mathrm{MEO}_{2} \mathrm{MA}\right)$ is related to the increasing of size distribution of the nanogels. If we compare the fluorescence emission of both type of nanogels, those based on the longer monomer, QD@SEOMA@pMEO $2 \mathrm{MA}$, exhibit the same behaviour but the fluorescence enhancement in dichloromethane (Table 1) is lower than for its homologous QD@SEMA@pMEO 2 MA nanogel. However, the higher polymer shell diameter of QD@SEOMA@pMEO 2 MA nanogel provides a better solubility in aqueous medium. On other hand, it should be noted that all the nanogels maintain their fluorescence emission properties in aqueous medium, with a significant shift toward the red (between $80-140 \mathrm{~nm}$ ) with respect to the dichloromethane solution, (as can be observed in Figure S4). Nevertheless, the nanogels do not show significant variation on the emission properties in aqueous solutions as well as in their hydrodynamic diameter by changing the $\mathrm{pH}$ of the medium.

A study of thermo- and $\mathrm{pH}$ - response in aqueous media was done with these materials. The monomer $\mathrm{MEO}_{2} \mathrm{MA}$ selected to build-up the polymeric shell leads to a promising new family of thermo-responsive polymers with easily tuneable LCST by copolymerization with methacrylic monomers with longer oligo(ethylene glycol) side chains. ${ }^{22-25}$ They undergo a drastic reversible collapse in water when the
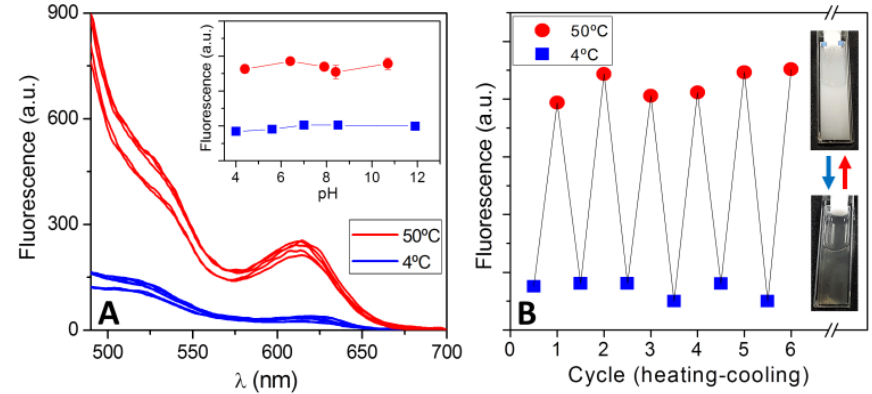

Figure 3. A) Fluorescence spectra emission of water-dispersable p(QD@SEOMA@pMEO $2 M A)$ nanogels in phosphate buffer at pH 4 at two temperatures. Inset: Evolution of the fluorescence with the temperature at different pHs (from 12 to 4). B) Emission evolution along different and consecutives cycles of cooling-heating. Inset: real photograph of p(QD@SEOMA@pMEO $\left.{ }_{2} M A\right)$ nanogels under and above the Tc.

temperature reached the temperature of collapse assumed in this work as the cloud point (Tc). The polymeric coating endowed the QDs nanohybrids with thermo-responsive properties as in aqueous solution they exhibited reversible aggregation/disaggregation above and below the Tc (Figure 2), respectively; reaching slightly higher values $\left(31.4 \pm 0.6^{\circ} \mathrm{C}\right)$ for QD@SEMA@pMEO 2 MA nanogels and similar values (25.9 \pm $1.7^{\circ} \mathrm{C}$ ) for QD@SEOMA@pMEO ${ }_{2} \mathrm{MA}$ nanogels in water at $\mathrm{pH} 7$ than $\mathrm{p}\left(\mathrm{MEO}_{2} \mathrm{MA}\right)$ homopolymer $\left(\mathrm{ca} .26 .5^{\circ} \mathrm{C}\right) .{ }^{23,}{ }^{24}$ These differences can be attributed to the effect of the polymer concentration on the temperature of aggregation; so that the hybrid with higher shell coating, QD@SEOMA@MEO ${ }_{2} M A$, presents higher polymer concentration that leads to a decreasing of the cloud temperature. Other remarkable difference between both systems is the $\mathrm{pH}$-response, thus QD@nanogels based on SEMA do not present significant variations of the Tc values with changes in the $\mathrm{pH}$ (Figure 2A). This fact indicates the low probability of free thiol groups in the final nanogel and so, a preferential orientation towards the surface of the nanoparticle. Meanwhile, the nanogels based on the longer monomer, SEOMA, show a slight dependence of the Tc with the $\mathrm{pH}$, decreasing until $22^{\circ} \mathrm{C}$ at acid $\mathrm{pH}$ (Figure 2B).

Moreover, we studied the effect of temperature and $\mathrm{pH}$ on the emissive properties of both water-dispersible hybrid nanogels. Two temperatures, one above $\left(50^{\circ} \mathrm{C}\right)$ and other below $\left(4^{\circ} \mathrm{C}\right)$ the $\mathrm{Tc}$ were selected and the nanogel emission was measured at different pHs. As despicts in Figure 3 and S5, the emissive properties of the nanohybrids depend on the aggregation state of the nanogels. In the whole range of $\mathrm{pHs}$ studied (from 12 to 4 ) the result obtained was very similar, observing a remarkable increase of the emission of fluorescence at high temperature, after the collapse of the nanogels. Nanogels emit at least 50\% more above the temperature of collapse with a significant increase of both emission peaks $\left(520 \mathrm{~nm}\right.$ and $615 \mathrm{~nm}$ ) at $50^{\circ} \mathrm{C}$ (Figure $3 \mathrm{~A}$ and $\mathrm{S} 5 \mathrm{~A})$. However, the $\mathrm{pH}$ of the medium has no evident effect on the emission properties of the nanogels at the studied temperatures. Both temperature and $\mathrm{pH}$ effects showed total reversibility after repetitive aggregation-disaggregation cycles (Figure 3B and S5B). Further characterization of the thermoresponsive properties of the nanogels was carried out by 
dynamic light scattering (DLS) and STEM. Variable temperature DLS measurements showed in both cases an average hydrodynamic diameter in number $\left(D_{h}\right)$ that rapidly increases by exceeding the collapse temperature (Figure 4).

The aggregation of the nanogels is caused because the protecting polymeric shell of the nanohybrid experiences a sudden decrease of solubility as function of temperature. So, the increase in the temperature causes the collapse of the nanogels forming large aggregate inter-particulate clusters independently of the $\mathrm{pH}$. Whereas the effect of $\mathrm{pH}$ is only observed when the collapsed SEOMA based nanogels are exposed to acidic $\mathrm{pH}$, the former aggregates become larger. The collapse is totally reversible when the samples are cooled again. This temperature is in accordance with the cloud point measured by UV. For both nanogels, the collapse occurs between $25-26^{\circ} \mathrm{C}$ and the average hydrodynamic diameter in number $\left(D_{h}\right)$ changes from few nanometers, at temperature bellow VPTT, to hundred namometers above VPTT under $\mathrm{pH} 7$ (from $4.4 \pm 0.7 \mathrm{~nm}$ to $380 \pm 130 \mathrm{~nm}$ ) for p(QD@SEMA@pMEO $2 \mathrm{MA}$ ) nanogels and from $8.6 \pm 1.0 \mathrm{~nm}$ to $917 \pm 176 \mathrm{~nm}$ for $\mathrm{p}\left(\mathrm{QD} @ S E O M A @ \mathrm{pMEO}_{2} \mathrm{MA}\right)$ nanogels. Figure $4 \mathrm{C}, 4 \mathrm{~F}$ and $\mathrm{S} 6$ depicts representative sTEM images of the two hybrid nanogels obtained, prepared from the same water colloidal solution, at temperatures below and above the cloud point. These images are in accordance with the DLS former results. Figure $4 \mathrm{C}$ and $4 \mathrm{~F}$ depicts how while at low temperature in all the $\mathrm{pH}$ range the nanogels appear totally dispersed without showing any type of collapse or aggregation; when the temperature of the medium increased above Tc, a clear aggregation was observed appearing larger structures (900$1100 \mathrm{~nm}$ ) in all range of $\mathrm{pH}$ studied, being even more drastic (reaching $\sim 4 \mu \mathrm{m}$ ) at acid $\mathrm{pHs}$ for SEOMA based nanogels. The accumulation of the nanogels at specific site by thermally induced precipitation could be very useful to facilitate imaging and treatment of tumors. ${ }^{36}$

\section{Synthesis and characterization of hybrid thermo-responsive hydrogels}

In order to ascertain if the new hybrid nanoparticles functionalized with the thiolated methacrylic monomers (QD@SEMA and QD@SEOMA) actually act as real centres of multi-crosslinking in the nanogels synthesis, we have also synthesized novel thermo-responsive hydrogel nanocomposites based on the copolymerization of this new hybrid nanoparticles with $\mathrm{MEO}_{2} \mathrm{MA}$, called as QD@SEMA$\mathrm{pMEO}_{2} \mathrm{MA}$ and QD@SEOMA-pMEO ${ }_{2} \mathrm{MA}$. So, redox bulk polymerization of $\mathrm{MEO}_{2} \mathrm{MA}$ was carried out in presence of different amounts of the multicrosslinker nanoparticles $(1,2$ and $3 \mathrm{wt} \%)$ and in absence of additional crosslinker as shown in Scheme 1.

Once obtained the macrogels, the impurities were removed by washing the obtained films with water. Films showed good consistency, and, no release of the fluorescent nanohybrids was detected in the wash medium, demonstrating that the nanohybrids are forming structural part of the hydrogels. Note that in absence of crosslinker
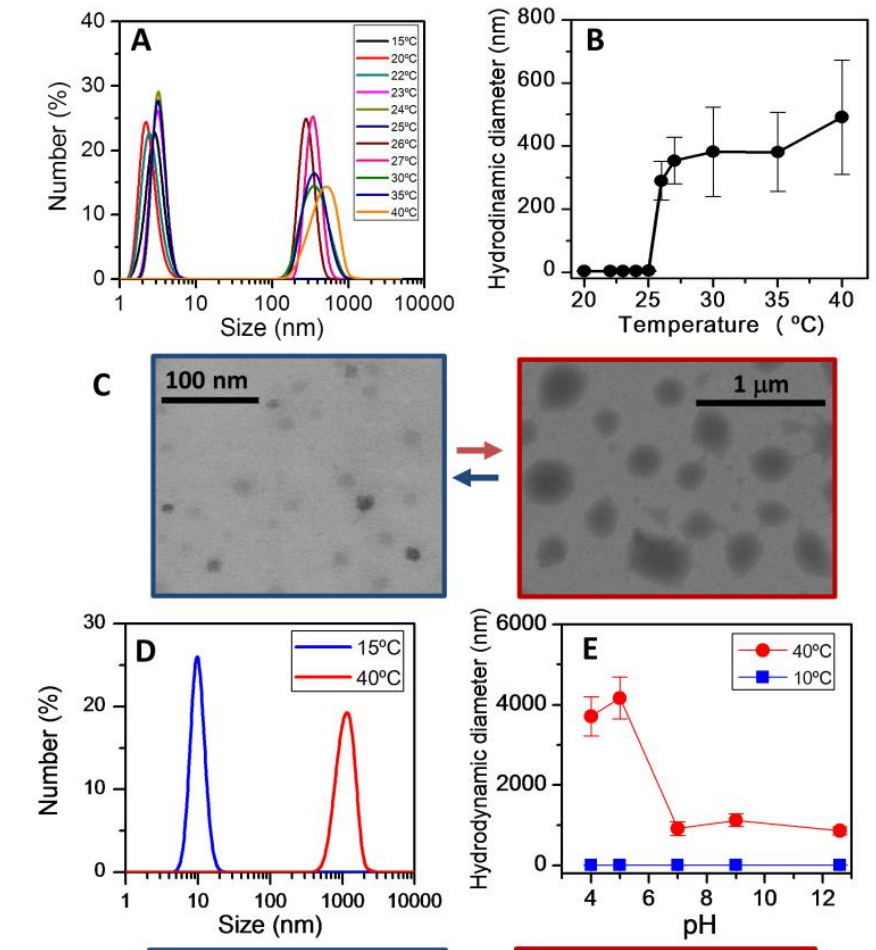

$\mathbf{F}$
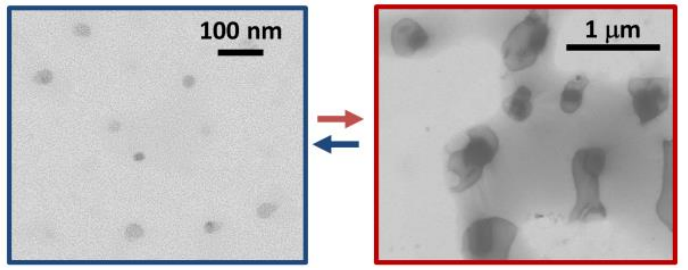

Figure 4. A, B) Dependence of hydrodynamic diameter versus temperature for nanogels of QD@SEMA@pMEO $\mathrm{MA}_{2}$ dissolved $\left(1 \mathrm{mg} \mathrm{mL}^{-1}\right)$ in water solution equilibrated to $\mathrm{pH} 7$. C) Representative sTEM images at $4^{\circ} \mathrm{C}$ and $50^{\circ} \mathrm{C}$; the $\mathrm{pH}$ of both colloidal samples were adjusted to $\mathrm{pH}$ 7. D) Dependence of the hydrodynamic diameter at $\mathrm{pH} 9$ at two temperatures ( 10 and $40^{\circ} \mathrm{C}$ ) for QD@SEOMA@ $\mathrm{pMEO}_{2} \mathrm{MA}$ nanogels. E) Dependence of the hydrodynamic diameter versus $\mathrm{pH}$ at two temperatures $\left(10\right.$ and $40^{\circ} \mathrm{C}$ ) for QD@SEOMA@pMEO 2 MA nanogels. F) Representative sTEM images at $4^{\circ} \mathrm{C}$ and $50^{\circ} \mathrm{C}$; the $\mathrm{pH}$ of both colloidal samples was adjusted to $\mathrm{pH}$ 9. All measured included the average of at least of ten measurements at each temperature.

nanoparticles the film is not produced because the $\mathrm{pMEO}_{2} \mathrm{MA}$ homopolymer was soluble in water.

First the swelling properties of the hybrid hydrogels were characterized in water as summarized in Table S2. Note that hydrogels with more than 2 wt\% of crosslinker nanoparticles of QD@SEMA or 3 wt\% of QD@SEOMA were synthesized but they show nanoparticles aggregation after polymerization and for this reason they were no longer studied. On the other hand, and contrary to what a priori might be expected, the equilibrium swelling increases with the content of hybrid nanoparticles based on SEOMA in the hydrogel, as shown in Table S2. This result indicates that the incorporation of these nanohybrids in the hydrogel influences the hydrophilic/hydrophobic balance of the final macrogel making the polymer more hydrophilic, and this effect exerts a greater influence on the swelling degree than the degree of crosslinking reached. On the other hand, these hydrogel nanocomposites are thermo-responsive showing changes in their equilibrium swelling with the temperature. So, an abrupt 


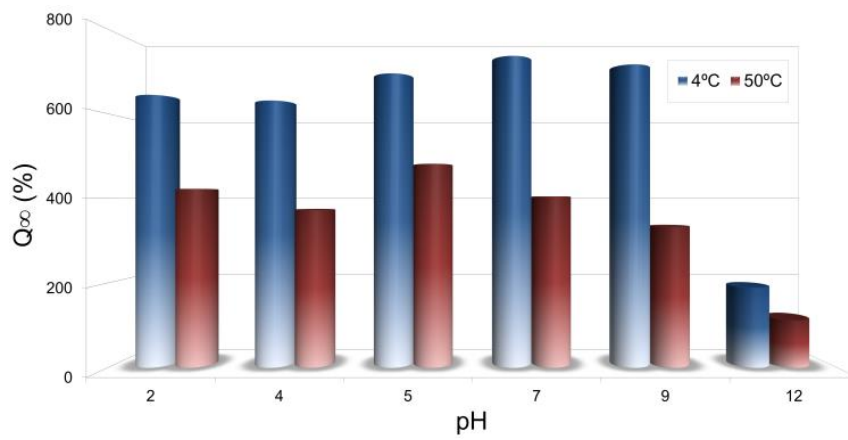

Figure 5. Variation of the equilibrium swelling $\left(\mathrm{Q}_{\infty}\right)$ of QD@SEOMA-pMEO $\mathrm{Q}_{2} \mathrm{MA}-2 \mathrm{wt} \%$ hydrogels reached as a function of the $\mathrm{pH}$ of the medium calculated at two temperatures $\left(4^{\circ} \mathrm{C}\right.$ and $\left.50^{\circ} \mathrm{C}\right)$.

change in volume and an increase in the opacity are observed above a critical temperature, called volume phase transition temperature (VPTT), in the same way that the LCST in the linear polymers. ${ }^{37,38}$

In all studied cases the increase in the temperature of the medium effectively causes the gel collapse, the expelling of water and consequently, a decrease of the swelling degree is observed as represented in the example of Figure 5. However the swelling degree at both above and below VPTT has not been practically affected by the $\mathrm{pH}$ excepting at highly basic medium where a drastic decrease is observed. The significant changes in the swelling of these hydrogels as function of temperature is accompanied by a sudden increase of the hydrogel opacity that permits to estimate the collapsing temperature (Tc) by the cloud point. These results for a representative sample, QD@SEOMA-pMEO 2 MA-3 wt\%, are presented in Figure 6A for different $\mathrm{pHs}$.

From these curves the temperatures of collapse (Tc) of the hydrogels were obtained as a function of $\mathrm{pH}$, observing that the same behaviour occurs in all cases, independently of the monomer nature, the $\mathrm{pH}$ of the medium or the amount of crosslinking NPs used in its synthesis. Only at $\mathrm{pH} 12$ no variations in absorbance were observed since the opacity is high throughout all the temperature range, however, the thermal response is notable for the remaining $\mathrm{pHs}$, especially at acid $\mathrm{pH}$, where the collapse temperature occurs around 22$24^{\circ} \mathrm{C}$. The $\mathrm{Tc}$ remains practically constant throughout the $\mathrm{pH}$ range studied except at $\mathrm{pH} 12$ as mentioned. This effect can be attributed to the thiolate/thiol balance of the monomer (SEMA or SEOMA). At higher $\mathrm{pH}$ there is a large amount of thiolate
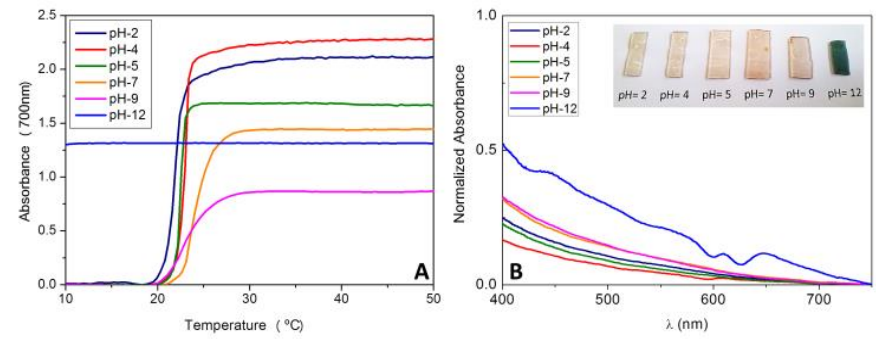

Figure 6. A) Variation of the absorbance at $700 \mathrm{~nm}$ versus temperature of hybrid hydrogels QD@SEOMA-pMEO 2 MA-3 wt\% as a function of $\mathrm{pH}$. B) Absorbance spectra recorded at low temperature $\left(10^{\circ} \mathrm{C}\right)$ of QD@SEOMA-pMEO ${ }_{2} \mathrm{MA}-3 \mathrm{wt} \%$ as a function of $\mathrm{pH}$. Inset: Real photographs of the hydrated hybrid hydrogels obtained as a function of the $\mathrm{pH}$ of the medium.
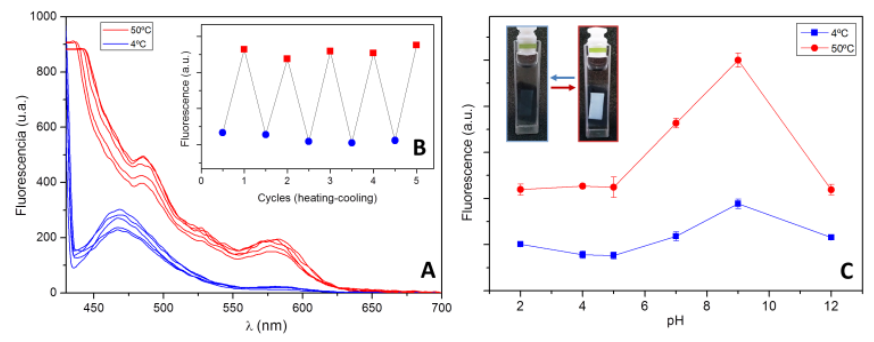

Figure 7. A) Fluorescence emission spectra and $\mathrm{B})$ its evolution during different and successive cycles of cooling $\left(4^{\circ} \mathrm{C}\right)$ and heating $\left(50^{\circ} \mathrm{C}\right)$ of hybrid hydrogel QD@SEOMA$\mathrm{pMEO}_{2} \mathrm{MA}-2 \mathrm{wt} \%$ submerged in phosphate buffer at $\mathrm{pH}-5$. C) Variation of fluorescence emission of the hydrogel as a function of the $\mathrm{pH}$ and temperature. Inset: real photographs of the same hydrogel recorded a two temperatures are included.

anions which give the hybrid a poor solubility that causes the loss of its thermosensitive character.

However, the most relevant result was obtained at high $\mathrm{pH}$ (ca. 12) when an abrupt change in the absorption spectrum was observed with the apparition of an intense peak at $650 \mathrm{~nm}$ (Figure 6B) as well as a considerable decrease in the degree of swelling with a sudden decrease in the VPTT $\left(c a .10^{\circ} \mathrm{C}\right)$. As can be seen in the attached picture this effect is also translated with a noticeable colour change in the hydrogel when it is maintained in conditions of basic $\mathrm{pH}$ turning from red to intense blue, as seen in the inset of Figure 6B. This colour response is notorious and independent of the crosslinker nature and its amount in the hydrogel and it is totally reversible and reproducible for all macrogels studied. At the moment we cannot give a plausible explanation to this phenomenon. The only experimental evidence found at this time is the change observed in the absorption spectrum of gels at $\mathrm{pH} 12$ (Figure 6), but this colour change is not associated with any other change observed in emission bands, only a decrease of the fluorescence intensity is observed. In previous work we reported that hydrogels bearing thiol groups exhibit $\mathrm{pH}$-responsiveness due to the ionization/protonation of thiol groups ${ }^{37}$ and this behaviour has also been previously described by other authors ${ }^{39,40}$, so a possible hypothesis seems to be probably related to both the strong polymer collapse at this alkaline $\mathrm{pH}$ (Figure 5) and the possible total ionization of thiol groups $(-\mathrm{SH})$ into thiolated groups $\left(-\mathrm{S}^{-}\right)$reached at strong alkaline $\mathrm{pH}$ that modifies the surface around the $\mathrm{QD}$ and change the surrounding of the functionalized nanoparticle. Previous groups have investigated the stability of colloidal nanocrystals coated with thiolate ligands upon $\mathrm{pH}$ change. ${ }^{41}$ The thiol group of a water-soluble ligand is deprotonated at high $\mathrm{pH}$, and thiolate has been shown to bind the QDs surface more strongly than thiol. ${ }^{42}$ They also discovered that the $Q Y$ could be improved by adding an appropriate reducing agent.

Finally, we detected how these hybrid hydrogels also experience a sudden change in their emission properties by the effect of temperature (Figure 7). In all cases, it has been observed, a remarkable increase in its emission of fluorescence after the collapse of the hydrogel (Figure 7A). This effect occurs at all pHs studied, being most notable at neutral-basic $\mathrm{pH}$ when SEOMA is used (Figure 7C), while for 
SEMA hybrid this effect is no affected by $\mathrm{pH}$ in the range studied (Figure S7).

The experiments are fully reproducible and repeated without giving rise to variations in the emission properties of the hydrogel after being subjected to several consecutive cooling-heating cycles, as shown in Figure 7A, B and Figure S7.

In comparative terms, the behavior of nanogels and hydrogels is similar in many respects, leaving aside the obvious differences due to their nanometric or macroscopic dimensions. Thus, both systems undergo a collapse accompanied by an increase in opacity at the VPTT of the polymer and, as it can be observed comparing Figures 2 and $6 \mathrm{~A}$, occur at similar temperature. In the case of nanogels the increase in opacity is due to the loss of the colloidal stability provided by the polymer shell at the transition temperature which leads to the formation of large, more stable aggregates. In the case of the hydrogel what happens is a decrease in volume and the separation of polymer/water phases is what gives rise to the opacity of the gel.

With regard to photoluminescence, this property is remarkable in both systems and both experiment the increase in the photoluminescence when increasing the temperature, although the red shift (relative to QD coated by the thiolated monomer) is greater in the case of the nanogels than in the macroscopic hydrogels.

Differences in dimensional scale will be important and necessary for possible applications. Thus, while the QDs confined in the nanogels will be of great application in biosensing, their macroscopic counterparts will be applicable in optoelectronic devices.

\section{Conclusions}

In summary, in this report we developed a new strategy to obtain hydrophilic luminescent and thermo-responsive QDs. In a first step, pristine hydrophobic QDs have been successfully functionalized with new methacrylic monomers bearing a protected thiol group capable of connecting with the surface of the nanoparticles. The methacrylic decorated QDs allow the production of new smart hybrid QD@nanogels and hydrogel nanocomposites based on $\mathrm{MEO}_{2} \mathrm{MA}$ acting as a multicrosslinking agent. Differences on the synthesis of both systems, thus joint to their different intrinsic structure, provide a wide range of potential applications in biomedicine and biotechnological fields.

In the synthesis of QD@nanogels a thin layer of $\mathrm{pMEO}_{2} \mathrm{MA}$, thermo-responsive polymer, growths over the multifunctional nanoparticle because of their reactive methacrylic groups, by suitable radical solution polymerization conditions. The new hybrid QD@nanogels kept nanometric size (6-8nm) and fluorescence emission properties, both in organic solvents and in aqueous media. In addition, these QD@nanogels experience size changes due to the collapse of the polymeric shell forming larger aggregates by the effect of temperature at the same time that a remarkable increase of fluorescence emission is detected. This aggregation behaviour with increasing temperature is potentially useful for local imaging and drug release.

On the other hand, the QD decorated with thiolmethacrylic-monomer allow their use as multicrosslinking agent in the synthesis of new hybrid hydrogels based on $\mathrm{pMEO}_{2} \mathrm{MA}$ with suitable mechanical properties, maintaining the photoluminescent of the starting NPs and also with response to external stimuli: $\mathrm{pH}$ and temperature of the medium. As it was observed for QD@nanogels, an important increase in fluorescence is detected with the collapse of the gels at all $\mathrm{pHs}$ studied. Also at very basic $\mathrm{pH}(\mathrm{pH}-12)$ the hydrogels undergo a colour change from red to fully reproducible blue, which makes these gels promising $\mathrm{pH}$ sensors. These new multifunctional hybrid materials present very promising expectations of applicability as biosensors in the biomedical field derived from the change of properties under certain environmental conditions (light, $\mathrm{pH}$ and temperature).

\section{Acknowledgements}

This work received financial support from the Ministerio de Economía y Competitividad (MINECO) from Spain through the Project MAT2014-57429-R. Also M. Liras thanks to MINECO and European Social Fund for a Ramón y Cajal contract (RyC2015-18677).

\section{References}

1. B. Sierra-Martin and A. Fernandez-Barbero, Soft Matter, 2015, 11, 8205-8216.

2. M. Molina, M. Asadian-Birjand, J. Balach, J. Bergueiro, E. Miceli and M. Calderon, Chemical Society Reviews, 2015, 44, 6161-6186.

3. J. J. Giner-Casares, M. Henriksen-Lacey, M. CoronadoPuchau and L. M. Liz-Marzán, Materials Today, 2016, 19, 19-28.

4. Y. Tao, M. Li, J. Ren and X. Qu, Chemical Society Reviews, 2015, 44, 8636-8663.

5. G. Lv, W. Guo, W. Zhang, T. Zhang, S. Li, S. Chen, A. S. Eltahan, D. Wang, Y. Wang, J. Zhang, P. C. Wang, J. Chang and X. J. Liang, ACS Nano, 2016, 10, 9637-9645.

6. R. Agarwal, M. S. Domowicz, N. B. Schwartz, J. Henry, I. Medintz, J. B. Delehanty, M. H. Stewart, K. Susumu, A. L. Huston, J. R. Deschamps, P. E. Dawson, V. Palomo and G. Dawson, ACS Chemical Neuroscience, 2015, 6, 494-504.

7. S. Hu, S. Zeng, B. Zhang, C. Yang, P. Song, T. J. Hang Danny, G. Lin, Y. Wang, T. Anderson, P. Coquet, L. Liu, X. Zhang and K.-T. Yong, Analyst, 2014, 139, 4681-4690.

8. K. G. Neoh and E. T. Kang, Polymer Chemistry, 2011, 2, 747-759.

9. S. Kango, S. Kalia, A. Celli, J. Njuguna, Y. Habibi and R. Kumar, Progress in Polymer Science, 2013, 38, 1232-1261.

10. X. Li, L. Sun, X. Wei, Q. Luo, H. Cai, X. Xiao, H. Zhu and K. Luo, J. Mater. Chem. B, 2017, 5, 2763-2774.

11. L. J. Hill, N. Pinna, K. Char and J. Pyun, Progress in Polymer Science, 2015, 40, 85-120.

12. B. Sarkar and P. Alexandridis, Progress in Polymer Science, 2015, 40, 33-62. 
13 M. Hood, M. Mari and R. Muñoz-Espí, Materials, 2014, 7, 28. 4057.

14. F. Zhang, E. Lees, F. Amin, P. Rivera_Gil, F. Yang, P. 29. Mulvaney and W. J. Parak, Small, 2011, 7, 3113-3127.

15. M. Liras, O. García, N. Guarrotxena, M. Palacios-Cuesta and I. Quijada-Garrido, Polymer Chemistry, 2013, 4, 57515759.

16. M. Liras, M. González-Béjar, E. Peinado, L. FrancésSoriano, J. Pérez-Prieto, I. Quijada-Garrido and O. García, Chem. Mater., 2014, 26, 4014-4022.

17. M. Liras, E. Peinado, P. Cañamero, I. Quijada-Garrido and O. García, J Polym Sci Part A, 2014, 52, 3087-3095.

18. M. Liras, I. Quijada-Garrido, M. Palacios-Cuesta, S. MuñozDurieux and O. García, Polymer Chemistry, 2014, 5, 433442.

19. N. Garcia-Bosch, M. Liras, I. Quijada-Garrido and O. Garcia, RSC Advances, 2016, 6, 67643-67650.

20. M. Liras, O. Garcia, I. Quijada-Garrido, G. Ellis and H. J. Salavagione, Journal of Materials Chemistry C, 2014, 2, 1723-1729.

21. A. Ferrández-Montero, I. Quijada-Garrido, M. Liras and O. García, European Polymer Journal, 2016, 84, 565-576.

22. N. Fechler, N. Badi, K. Schade, S. Pfeifer and J. F. Lutz, Macromolecules, 2009, 42, 33-36.

23. J.-F. Lutz, J. Polymer Sci. Part A: Polymer Chem., 2008, 46, 39. 3459-3470.

$24 . \quad J$. F. Lutz, A. Hoth and K. Schade, Des. Monomers Polym., 2009, 12, 343-353

25. J.-F. Lutz and A. Hoth, Macromolecules, 2005, 39, 893896.

26.

L. Rene-Boisneuf and J. C. Scaiano, Chem. Mater., 2008, 20, 6638-6642.

27. W. W. Yu, L. Qu, W. Guo and X. Peng, Chem. Mater., 2003, 15, 2854-2860.
K. Rurack and M. Spieles, Analytical Chem., 2011, 83, 1232-1242.

D. Magde, R. Wong and P. G. Seybold, Photochemistry and Photobiology, 2002, 75, 327-334.

30. F. Gao, P. Bajwa, A. Nguyen and C. D. Heyes, ACS Nano, 2017, 11, 2905-2916.

31. A. J. Morris-Cohen, M. Malicki, M. D. Peterson, J. W. J. Slavin and E. A. Weiss, Chem. Mater., 2013, 25, 11551165.

32. S. F. Wuister, C. de Mello Donegá and A. Meijerink, The Journal of Physical Chemistry B, 2004, 108, 17393-17397.

33. Y. Zhang and A. Clapp, Sensors, 2011, 11, 11036.

34. J. Aguilera-Sigalat, S. Rocton, J. F. Sanchez-Royo, R. E. Galian and J. Perez-Prieto, RSC Advances, 2012, 2, 16321638.

35. A. Sukhanova, J. Devy, L. Venteo, H. Kaplan, M. Artemyev, V. Oleinikov, D. Klinov, M. Pluot, J. H. M. Cohen and I. Nabiev, Analytical Biochemistry, 2004, 324, 60-67.

36. H. He, X. Zheng, J. Zhang, S. Liu, X. Hu and Z. Xie, Journal of Materials Chemistry B, 2017, 5, 2491-2499.

M. P. Montero-Rama, M. Liras, O. García and I. QuijadaGarrido, European Polymer Journal, 2015, 63, 37-44.

N. Badi and J. F. Lutz, J. Control. Release, 2009, 140, 224229.

M. Bajomo, J. H. G. Steinke and A. Bismarck, The Journal of Physical Chemistry B, 2007, 111, 8655-8662.

R. Jayakumar, R. L. Reis and J. F. Mano, Drug Delivery, 2007, 14, 9-17.

J. Aldana, N. Lavelle, Y. Wang and X. Peng, Journal of the American Chemical Society, 2005, 127, 2496-2504.

P. Schapotschnikow, B. Hommersom and T. J. H. Vlugt, The Journal of Physical Chemistry C, 2009, 113, 1269012698. 